\title{
Estimation of Ground-Glass Opacity Measurement in CT Lung Images
}

\author{
Yuanjie Zheng $^{1}$, Chandra Kambhamettu ${ }^{1}$, Thomas Bauer ${ }^{2}$, and Karl Steiner ${ }^{3}$ \\ ${ }^{1}$ Department of Computer Science, University of Delaware, Newark, DE USA \\ ${ }^{2}$ Helen F. Graham Cancer Center, Christiana Care Health Services, \\ Newark, DE USA \\ ${ }^{3}$ Delaware Biotechnology Institute, University of Delaware, Newark, DE USA
}

\begin{abstract}
We propose to measure quantitatively the opacity property of each pixel in a ground-glass opacity tumor from CT images. Our method results in an opacity map in which each pixel takes opacity value of [0-1]. Given a CT image, our method accomplishes the estimation by constructing a graph Laplacian matrix and solving a linear equations system, with assistance from some manually drawn scribbles for which the opacity values are easy to determine manually. Our method resists noise and is capable of eliminating the negative influence of vessels and other lung parenchyma. Experiments on 40 selected CT slices of 11 patients demonstrate the effectiveness of this technique. The opacity map produced by our method is invaluable in practice. From this map, many features can be extracted to describe the spatial distribution pattern of opacity and used in a computer-aided diagnosis system.
\end{abstract}

\section{Introduction}

With recent advances in lung cancer screening based on computed tomography (CT), the detection of small cancers has been notably increasing [1]. The main goal of the CT based detection is to improve the survival rate by detecting small cancers when they are early in their biologic evolution for lung radiotherapy.

Ground-glass opacity (GGO) is a highly significant finding on high-resolution CT because it is associated with different kinds of lung diseases such as adenocarcinomas 2, bronchioloalveolar carcinoma 3, idiopathic pulmonary fibrosis and sarcoidosis 4, etc. GGO is defined as hazy increased CT attenuation of the lung without entire obscuration of the lung parenchyma within it. In contrast, a solid nodule completely obscures the entire lung parenchyma within it. Lung tumor may also appear as a mixture of GGO and solid nodules. The phenomenon of GGO and solid nodules in the CT image is caused by the different physical densities of the lung tissues. Much higher frequency of malignancy was shown in lung tumor with GGO 5]. Moreover, it was reported that GGO percentage (the proportion of GGO area in a lung tumor) [26] and GGO spatial distribution pattern 4] are the significant prognostic factors of some lung diseases. Therefore, measuring the opacities of the lung tumor pixels with CT image is vital to the radiologic analysis of a lung cancer candidate. 
There exist basically three kinds of measuring methods of GGO, but all of them are limited in practice. The first way, possibly the one being most popularly used, is the visual assessment. The severity of GGO is usually classified subjectively as none, mild, moderate or severe. This method suffers from its low precision, inter-reader variability, and inability to give quantitative assessment. The second kind of methods 26] proposed recently were designed to provide objective measurement of GGO percentage. They use two CT window levels to show the CT image, and segment the tumor areas and solid nodule area by thresholding the displayed images with some public software such as the Scion Image 6] and the NIH image software [2]. The GGO area is then computed as the tumor area subtracted by the solid nodule area and the GGO percentage is defined as the percentage of the GGO area in the tumor area. Although these methods are capable of eliminating the inter-reader variability, they cannot analyze the GGO spatial distribution pattern because they are not able to provide the quantitative opacity value of each tumor pixel. In addition, the accuracy of the computed tumor area and solid nodules' area highly depend on the specification of level value of CT windows. Another problem of these methods is that they cannot resist noise, and need extra processes to suppress vessel appearance within the tumor, which appears very similar to solid nodules. The last kind of method for measuring GGO uses the probability density function (pdf) 78. The pdf was proved to be capable of distinguishing GGO and other lung parenchyma, hence being widely used in GGO detection and segmentation. However, only few papers can be found that provide quantitative measurement of the opacity of GGO pixels. Besides the three existing methods, we also noticed that the attenuation value in $\mathrm{CT}$ imaging for each GGO pixel can reflect in some degree the opacity, since the CT attenuation value has a linear relationship with the physical density of the tissue. However, measuring GGO on the basis of attenuation value alone is incomplete and subjective. Lung parenchyma may also be in the attenuation range of GGO.

In this paper, we propose an interactive scheme to measure quantitatively the per pixel opacity value of the lung GGO nodule in a 2D CT image. By manually inputting some scribbles for which the opacity values are easily determined by the user, our scheme can produce an accurate opacity map in which each pixel takes opacity value between [0-1]. The computation of the opacity map is accomplished efficiently by constructing a graph Laplacian matrix and solving a linear system of equations. Our method is resistant to noise and capable of eliminating the adverse affects of vessels or other lung parenchyma. Experiments on 40 selected CT slices of 11 patients demonstrate the effectiveness of our technique.

\section{Method}

We assume that a given CT lung slice $\mathcal{I}$ is a linear combination of a solid nodule layer $\mathcal{N}$ and a non-nodule layer $\mathcal{L}$ with an opacity map $\rho$ :

$$
\mathcal{I}(i)=\rho(i) \mathcal{N}(i)+(1-\rho(i)) \mathcal{L}(i), \quad i \in \Omega
$$


where $i$ indexes the pixels in $\mathcal{I}$ 's domain $\Omega$, and $\rho$ can be any value between $[0-1]$. In this equation, $\mathcal{I}$ is the given $\mathrm{CT}$ attenuation value, $\mathcal{N}$ represents the attenuation value by the nodule tissue, and $\mathcal{L}$ describes the attenuation value by lung parenchyma (such as vessel, bronchi, etc.), the air, and other non-nodule tissues in lung. We can imagine that $\mathcal{I}$ is formed by overlapping $\mathcal{N}$ on $\mathcal{L}$. For a solid nodule pixel, we expect $\rho(i)=1$, such that $\mathcal{I}(i)=\mathcal{N}(i)$, meaning that the layer $\mathcal{N}$ completely blocks $\mathcal{L}$. For a non-nodule pixel, we expect $\rho(i)=0$, such that $\mathcal{I}(i)=\mathcal{L}(i)$, meaning that the layer $\mathcal{N}$ is totally transparent. For a GGO pixel, we expect $0<\rho(i)<1$, such that the layer $\mathcal{N}$ is transparent and $\mathcal{I}(i)$ is the combination of $\mathcal{N}(i)$ and $\mathcal{L}(i)$. Moreover, the smaller the $\rho$, the more transparent the layer $\mathcal{N}$ is. We can see that $\rho$ in equation (1) is an efficient measurement of GGO opacity.

Given a CT lung slice $\mathcal{I}$, to compute the opacity map $\rho$ with equation (1), both the nodule layer value $\mathcal{N}$ and the non-nodule layer value $\mathcal{L}$ need to be simultaneously estimated for every pixel. Therefore, it is inherently an under-constrained problem because there are $3 T$ unknowns but $T$ equations if we assume the number of pixels in $\Omega$ is $T$.

To make the problem in equation (11) solvable, we add two constraints. The first constraint is enforced by setting some pixels, denoted by a set $S$, to some known values in the opacity map. These pixels are specified by manually drawing some scribbles on pixels for which the opacity values can be easily recognized by the user, for example, the solid nodule pixels (entirely formed by $\mathcal{N}$ and with $\rho$ value 1 ) and the pure non-GGO pixels (entirely formed by $\mathcal{L}$ and with $\rho$ value 0$)$. The pure non-GGO pixels can be the pure "air", "vessel" or other lung parenchyma. The second constraint is the locally spatial smoothness of the $\rho$ map. It is a neighborhood constraint to make neighboring pixels have similar $\rho$ values. It is based on the fact that the densities of the lung parenchyma usually show a spatially gradual change.

We notice that the similarities between the problem raised in this paper and the recent computer vision techniques in image matting 910] and soft segmentation [1]. We apply a technique similar to [9] for solving the opacity map in equation (1). Without computing $\mathcal{N}$ and $\mathcal{L}$, we estimate the opacity map $\rho$ by casting the problem as constructing a graph Laplacian matrix and solving a linear equations system. Given a CT slice data, a weighted graph is first formed for the graph Laplacian matrix and then the linear equations system is built upon the two constraints mentioned above.

\subsection{Specifying Pixels Interactively with an Interface}

By setting scribbles with an interactive interface, we manually specify some pixels which are not the mixture of $\mathcal{N}$ and $\mathcal{L}$, instead, are entirely formed by $\mathcal{N}$ or $\mathcal{L}$, as shown in Figure 1, For the specified pixels, we already know their opacity values, and use them as the first constraint to solve the opacity map with equation (1). Through our experiments, we found that the user can always very easily recognize some pixels near the tumor, which are entirely formed by $\mathcal{L}$. In addition, we found that for most of the tumors in our data sets the user 
can recognize some solid nodule areas. For the rare case when the whole tumor is ground-glass, the interface can facilitate the input from user's quantitative estimation of the opacity values $([0-1])$ for some pixels. For this case, the accuracy of the resulting opacity map depends on the user's initial estimation.

The interface shows the scribbles for $\mathcal{N}$ and $\mathcal{L}$ in different colors (as shown in Figure 10, and provides the freedom to the user to delete or add some scribbles. It also offers the flexibility to change the width of the scribble in the scribblesetting process. All the functions help the user produce good results.

\subsection{Solving for Opacity Map with Graph Laplacian Matrix}

We consider the given slice of CT data $\mathcal{I}$ as a graph $G$. The graph is defined as a pair $G=(V, E)$ with vertices $v \in V$ representing the pixels in the CT data and edges $e \in E \subset V \times V$ connecting two vertices. The number of vertices in $G$ is same as the number of pixels in $\Omega$, i.e. $T$. We define the set $N(i)$ of neighboring vertices of a vertex $i$ as the ones connected with $i$. For a given $\mathrm{CT}$ slice data $\mathcal{I}$ which is a data set in 2D grid, we construct its graph by connecting the pixels which are 4 -connected neighbors in the $2 \mathrm{D}$ grid. If we assign a weight $w_{i j}$ to each $e_{i j}$ connecting the vertices $i$ and $j$ to measure their connection, $G$ is called a weighted graph. Also, the degree of a vertex is defined as $d_{i}=\sum_{j \in N(i)} w_{i j}$. Then we can construct a diagonal matrix $D$ of size $T \times T$ for which $D_{i i}=d_{i}$ and $D_{i j}=0$ if $i \neq j$, and a matrix $W$ of size $T \times T$ for which $W_{i j}=w_{i j}$. Note that $w_{i i}=0$ for any $i$, and $W$ is symmetric if we set $w_{i j}=w_{j i}$. A matrix $L$ is then defined as

$$
L=D-W
$$

which is called graph Laplacian matrix. Note that $L$ is a sparse and symmetric matrix.

To accomplish the second constraint mentioned above for solving the opacity map, i.e. the spatially local smoothness of the $\rho$ value, we assume that $\rho$ value of an arbitrary pixel is the weighted average of the $\rho$ values in its neighboring pixels, and estimate $\rho$ by minimizing the below function

$$
E(\rho)=\sum_{i \in \Omega}\left(d_{i} \rho(i)-\sum_{j \in N(i)} w_{i j} \rho(j)\right)^{2} .
$$

If we concatenate the $\rho$ value of the pixels in $\Omega$ as a vector $\bar{\rho}$ of length $T$, equation (3) can be rewritten as

$$
E(\rho)=\bar{\rho}^{T} L \bar{\rho}
$$

where the graph Laplacian matrix $L$ is formed given a CT slice data $\mathcal{I}$ with the method talked above.

Note that the first constraint for solving the opacity map $\rho$ using equation (1) is the manual specification of a set of pixels $S$. For each element $i$ in $S$, we use $\rho^{\prime}(i)$ to denote its already known opacity value. 
The computation of $\rho$ with equation (11) by adding the two constraints mentioned above is expressed as below

$$
\rho=\arg \min \bar{\rho}^{T} L \bar{\rho}, \text { s.t. } \rho(i)=\rho^{\prime}(i), \forall i \in S .
$$

If we know all weight values in equation (3), equation (5) is an optimization of a quadratic problem with constraints. It can be solved using a number of standard methods such as the singular value decomposition (SVD). In this paper, we use a technique similar to 911, which has been shown to be efficient in image matting and soft segmentation. It is detailed below.

We partition $V$ in the graph $G$ of the given CT slice data into two subsets, $S$ denoting the specified pixels and $U$ representing the unspecified pixels. We can assume that the pixels in equation (5) have already been ordered such that pixels in $S$ are first and pixels in $U$ are second. Therefore, we can decompose the quadratic term in equation (5) into,

$$
\bar{\rho}^{T} L \bar{\rho}=\left[\begin{array}{c}
\bar{\rho}_{s} \\
\bar{\rho}_{u}
\end{array}\right]^{T}\left[\begin{array}{cc}
L_{s} & B \\
B^{T} & L_{u}
\end{array}\right]\left[\begin{array}{c}
\bar{\rho}_{s} \\
\bar{\rho}_{u}
\end{array}\right]=\bar{\rho}_{s}^{T} L_{s} \bar{\rho}_{s}+2 \bar{\rho}_{u}^{T} B^{T} \bar{\rho}_{s}+\bar{\rho}_{u}^{T} L_{u} \bar{\rho}_{u}
$$

where $\overline{\rho_{s}}$ and $\overline{\rho_{u}}$ are the vectors concatenated by the $\rho$ values of the pixels in $S$ and $U$ respectively. Note that we already know the values of $\bar{\rho}_{s}$ but not of $\bar{\rho}_{u}$. By differentiating the above equation with respect to $\bar{\rho}_{u}$ and setting it zero, we get

$$
L_{u} \bar{\rho}_{u}=-B^{T} \bar{\rho}_{s} .
$$

Equation (7) represents a system with $|U|$ linear equations and $|U|$ unknowns, and the matrix $L_{u}$ is sparse, symmetric, positive-definite, and guaranteed to be non-singular for a connected graph [9] as for our case. Therefore, we can get a unique solution for $\bar{\rho}_{u}$. By concatenating the $\bar{\rho}_{s}$ which is already known and $\bar{\rho}_{u}$ that is being computed with equation (7), we finally get the opacity map $\bar{\rho}=\left[\begin{array}{l}\bar{\rho}_{s} \\ \bar{\rho}_{u}\end{array}\right]$.

$L_{u}$ may seem huge because $|U|$ is possibly very large. However, in practice $L_{u}$ would not occupy too much memory space considering the fact that it is a very sparse matrix.

\subsection{Determining the Edge Weight of the Graph}

To solve the opacity map using equation (7), we need to know the weight values (shown in equation (3)) for the edges connecting two vertices in the graph. There are different ways to compute the weight. A very simple way is based on the squared difference between the two CT attenuation values and expressed as $w_{i j}=\exp \frac{-(\mathcal{I}(i)-\mathcal{I}(j))^{2}}{2 \sigma^{2}}$ where $\sigma$ is a constant. However, to produce better results, we employ the weight proposed in [10].

The weight in [10] was proposed by assuming the two layers $\mathcal{N}$ and $\mathcal{L}$ being locally smooth. We omit the derivation of the weight and only put the theoretical 


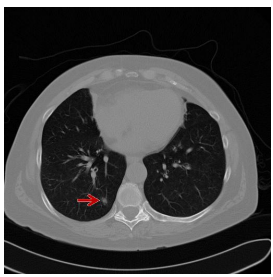

Slice 1

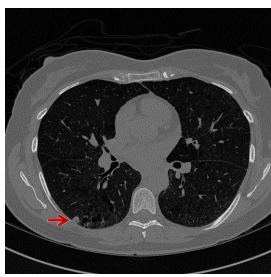

Slice 2

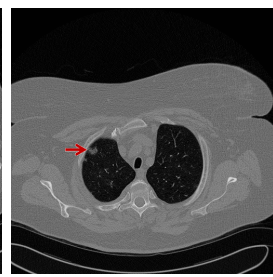

Slice 3

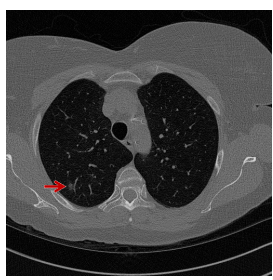

Slice 4
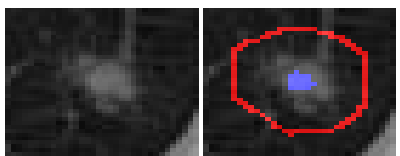

Slice 1

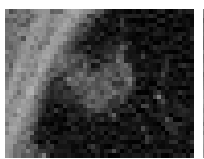

Slice 3
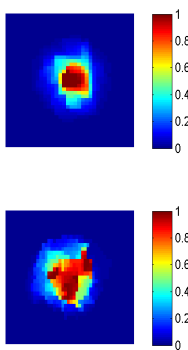

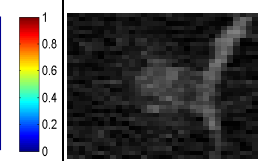

1
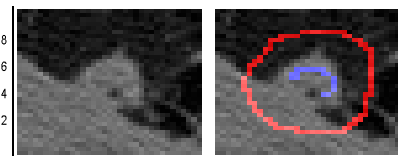

Slice 2

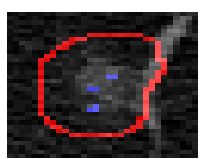

Slice 4
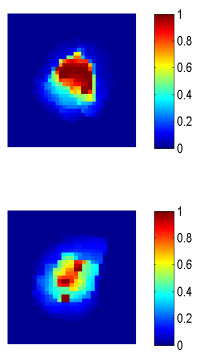

Fig. 1. Results of measuring ground-glass opacity map of tumors in 4 CT lung slices by our method. Upper most: the original CT images. Lower: the results by our method. For each slice, from left to right, zoom-in local patch containing the tumor, scribbles (blue color for foreground and red for background), and computed opacity map, respectively.

result below. For more details, the reader is referred to [10]. A weight $w_{i j}$ is computed based on all the local windows containing pixels $i$ and $j$ in $\Omega$. We set the window size to $3 \times 3$ in this paper. Denoting a local window by $\mathcal{W}_{k}$ where $k$ indexes the window, the weight is

$$
w_{i j}=\sum_{k \mid\{i, j\} \in \mathcal{W}_{k}}\left(\delta_{i j}-\frac{1}{\left|\mathcal{W}_{k}\right|}\left(1+\frac{\left(\mathcal{I}(i)-\mu_{k}\right) *\left(\mathcal{I}(j)-\mu_{k}\right)}{\epsilon /\left|\mathcal{W}_{k}\right|+\sigma_{k}^{2}}\right)\right)
$$

where $\delta_{i j}$ is the Kronecker delta taking value 1 when $i=j$ and 0 otherwise, $\mu_{k}$ and $\sigma_{k}$ are the mean and variance of the $\mathrm{CT}$ attenuation values of the pixels in the window $\mathcal{W}_{k}$, and $\epsilon$ is a regularization coefficient set to $10^{-5}$ in this paper.

\section{Results}

To test our GGO measurement method, we collected 40 slices from the 3D CT lung data sets of 11 patients. Each slice was chosen with such a way that there is a dominant tumor area. The images were scanned by a Siemens machine, with processed image size as $512 \times 512$ and the pixel resolution is $0.59 \mathrm{~mm} \times$ $0.59 \mathrm{~mm}$. Histologic diagnoses were made for all the patients by radiologists, and the histologic types include bronchioloalveolar carcinoma, adenocarcinoma and idiopathic pulmonary fibrosis, etc. 
Through experiments, we found that the user can very easily and quickly obtain an accurate opacity map for a GGO tumor using our techniques by simply drawing some scribbles. Trained user can get satisfactory opacity map for a GGO tumor within 15 seconds using a modern computer. Some results of the computed opacity maps for $4 \mathrm{CT}$ lung slices are shown in Figure 1.

We can also see two other prominent advantages of our method from the results in Figure 1, First, our method can provide an opacity map for a GGO tumor in which each pixel has an opacity value between [0-1]. To compute the GGO percentage [26], we can simply threshold the opacity map, and more importantly, we can set one threshold for different opacity maps. Second, our method resists noise and produces accurate opacity map even when the GGO tumor clings to thorax or is mixed with vessels. It is known that the attenuation value of a solid nodule pixel in a GGO tumor can be very similar to thorax and vessel. Regardless of the adverse affects of thorax and vessels which always bring in difficulties in GGO detection and segmentation, our method produced accurate results as shown in Figure 1

In addition, we obtained quantitative evaluations by thresholding (with threshold value 0.24 ) the opacity map, and comparing with the manual segmentation by a trained rater and with the refined manual segmentations using a graph cut based technique similar to [12]. The distance between two segmentation contours was used to measure the difference, which is defined as the average of each pixels shortest distance to the other contour. Based on all our test samples, the mean and standard deviation of these distances were found to be 4.2 and 4.9 pixels for the manual segmentation, and 3.4 and 4.3 pixels for the refined segmentation.

The opacity map computed by our method is extremely useful in practice. We can design different features to describe the spatial distribution pattern of the opacity values of the corresponding GGO tumor and use them in a computeraided diagnosis (CAD) system of lung cancer. To the best of our knowledge, our method is the only work to compute the quantitative opacity map for GGO lung tumors, hence, we have not performed comparisons with other techniques.

\section{Conclusions and Future Work}

We estimate an opacity map for GGO tumor in CT lung images to quantitatively measure the opacity properties. Given a CT image, some scribbles are drawn, and the opacity map is then estimated through constructing a graph Laplacian matrix and solving a linear equations system.

We believe that the opacity map computed with our method is invaluable in practice. Our method offers the quantitative assessment of per-pixel opacity; the radiologist can evaluate the GGO severity with high confidence. More importantly, we can extract different features describing the opacity map and use them in a CAD system. As the percentage of the GGO area within a tumor area is essential for diagnosis of several lung diseases [2 6], we believe that the features extracted from the opacity map will be extremely useful in a CAD system. Our future work will focus on extracting features from the opacity map and applying 
them to lung tumor diagnosis. These features can represent the statistics of the opacity values such as mean and variance etc., or describe the spatial distribution of the opacity values such as the moment invariants and Gabor textures.

\section{Acknowledgement}

This publication was made possible by Grant Number 2 P20 RR016472-08 under the INBRE program of the National Center for Research Resources (NCRR), a component of the National Institutes of Health (NIH).

\section{References}

1. Sobue, T., et al.: Screening for lung cancer with low-dose helical computed tomography: Anti-lung cancer association project. Journal of Clinical Oncology 20, 911-920 (2002)

2. Nakata, M., Sawada, S., Yamashita, M., Saeki, H., Kurita, A., Takashima, S., Tanemoto, K.: Objective radiologic analysis of ground-glass opacity aimed at curative limited resection for small peripheral non-small cell lung cancer. Journal of Thoracic and Cardiovascular Surgery 129, 1226-1231 (2005)

3. Jang, H.J., Lee, K.S., Kwon, O.J., Rhee, C.H., Shim, Y.M., Han, J.: Bronchioloalveolar carcinoma: focal area of ground-glass attenuation at thin-section ct as an early sign. Radiology 199, 485-488 (1996)

4. Engeler, C.E., Tashjian, J.H., Trenkner, S.W., Walsh, J.W.: Ground-glass opacity of the lung parenchyma: a guide to analysis with high-resolution ct. American Journal of Roentgenology 160, 249-251 (1993)

5. Henschke, C.I., Yankelevitz, D.F., Mirtcheva, R., McGuinness, G., McCauley, D., Miettinen, O.S.: CT screening for lung cancer: Frequency and significance of partsolid and nonsolid nodules. Am. J. Roentgenol. 178, 1053-1057 (2002)

6. Matsuguma, H., et al.: Objective definition and measurement method of groundglass opacity for planning limited resection in patients with clinical stage IA adenocarcinoma of the lung. European Journal of Cardio-thoracic Surgery 25, 1102-1106 (2004)

7. Zhao, B., Gamsu, G., Ginsberg, M.S., Jiang, L., Schwartz, L.H.: Automatic detection of small lung nodules on CT utilizing a local density maximum algorithm. Journal of Applied Clinical Medical Physics 4, 248-260 (2003)

8. Zhou, J., Chang, S., Metaxas, D.N., Zhao, B., Schwartz, L.H., Ginsberg, M.S.: Automatic detection and segmentation of ground glass opacity nodules. In: Larsen, R., Nielsen, M., Sporring, J. (eds.) MICCAI 2006. LNCS, vol. 4190, pp. 784-791. Springer, Heidelberg (2006)

9. Grady, L., Schiwietz, T., Aharon, S., Westerman, R.: Random walks for interactive alpha-matting. In: VIIP 2005 (2005)

10. Levin, A., Lischinski, D., Weiss, Y.: A closed form solution to natural image matting. In: CVPR 2006 (2006)

11. Grady, L.: Random walks for image segmentation. IEEE Transactions on Pattern Analysis and Machine Intelligence 28, 1768-1783 (2006)

12. Zheng, Y., Baloch, S., Englander, S., Schnall, M.D., Shen, D.: Segmentation and classification of breast tumor using dynamic contrast-enhanced MR image. In: Ayache, N., Ourselin, S., Maeder, A. (eds.) MICCAI 2007, Part II. LNCS, vol. 4792, pp. 393-401. Springer, Heidelberg (2007) 